\title{
Editorial
}

\section{Improvement by death in immunity}

\author{
SJ Martin ${ }^{\star 1}$ \\ Cell Death and Differentiation (2008) 15, 221-222. doi:10.1038/sj.cdd.4402293
}

Hector Munro, the English satirist and author, is credited with the somewhat unkind, but delicious, quotation 'he is one of those people who would be enormously improved by death'. While that comment may be somewhat harsh when applied to a particularly stubborn referee, for example, it is certainly appropriate when applied to the immune system, where the ability to control the death of its cellular constituents results in an immense improvement in the specificity and control of immune responses.

Although cell death control is clearly important in numerous biological systems, ${ }^{1}$ it is especially so within the immune system where regulated cell death plays a particularly pervasive and important role. Programmed cell death is initially used as a quality control measure during the development of $\mathrm{T}$ and $\mathrm{B}$ cells, whereby developing lymphocytes that do not meet the desired specifications are killed. ${ }^{2}$ This is to ensure that mature lymphocytes meet certain strict criteria, a major one being that they should not recognize and respond to 'self', before being released into the peripheral circulation. A second consideration, but no less important, is that the antigen receptors present on the surface of lymphocytes should actually work and be capable, at least in principle, of detecting non-self peptides which may represent infectious agents. While the programmed elimination of cells is used to sculpt the final form of most developing organs and tissues, ${ }^{1}$ the consequences of a failure to delete $a$ few cells here and there is likely to be relatively insignificant in most situations. However, because apoptosis is invoked to eliminate auto-reactive (i.e. directed against 'self') $T$ and $B$ lymphocytes during the development of the adaptive immune system, the escape of even a few such cells could eventually lead to full-blown autoimmunity. Thus, the processes of positive and negative selection of lymphocytes by apoptosis are fundamental to the generation of functional adaptive immune systems. Such is the stringency of these selection processes that it has been estimated that up to $98 \%$ of developing $T$ lymphocytes typically fail to pass the series of functional tests imposed upon these cells during development and are thus eliminated via apoptosis.

Improvement by death within the immune system does not stop there however. Programmed cell death is subsequently employed to ensure that peripheral, mature, lymphocyte numbers are maintained within certain limits. ${ }^{3,4}$ Because antigen-specific lymphocytes embark upon a dramatic expansion of their numbers upon encounter with appropriate antigen, this poses a potential problem. What to do with these cells after the infectious agent has been cleared from the body? While the retention of some of these cells is very useful insurance against a subsequent encounter with the same antigen-and indeed having more cells the second time around is the basis for immunological 'memory'-the old adage 'too much of a good thing' can also apply. The lymphocyte compartment, much like any other cell compartment in the body, is of a relatively defined size. ${ }^{5}$ Thus, a surfeit of particular antigen-specific lymphocyte populations can have the rather undesirable consequence of leading to a deficit of others. This could lead to gaps in the immune repertoire that could lead to vulnerability to particular infectious agents. Furthermore, because of ongoing daily encounter with new and old antigens, it is logical that controls have to be placed upon the numbers of lymphocytes that are allowed to survive each round of antigen-driven expansion. Without such controls, lymphocyte numbers would undoubtedly creep inexorably upwards, as successive pathogens are encountered, and would eventually become overwhelming in the way that leukemias and other malignancies do. Thus, in addition to acting as a quality control mechanism, apoptosis is also critical for homeostatic control of lymphocyte numbers and peripheral tolerance within the immune system. ${ }^{3-5}$ The solution to the continuous expansion problem is that recently expanded lymphocyte populations soon undergo apoptosis to restore their numbers to slightly above where they started. ${ }^{3-5}$ Interestingly, although it was thought for many years that peripheral deletion was controlled primarily by regulating apoptosis of lymphocyte populations directly, recent evidence suggests that programmed cell death of dendritic cells, the specialized cells that present antigen to lymphocytes, plays a key role here also. ${ }^{6}$

Quite apart from the role that programmed cell death plays in shaping and maintaining the immune repertoire, the ability of immune cells to invoke apoptosis in other cell types, due to such cells being infected by intracellular pathogens or displaying aberrant peptides, is also critical to the proper functioning of the immune system. ${ }^{7,8}$ Specialized cells, cytotoxic $T$ cells (CTLs) and natural killer (NK) cells have evolved as apoptosis-inducing cell types that cooperate to kill infected target cells. Indeed, much of the raison d'etre of the immune system revolves around killing; either the unwanted guests themselves, or cells that have been infected by such intruders. CTL and NK cells are also involved in refining

\footnotetext{
${ }^{1}$ Molecular Cell Biology Laboratory, Department of Genetics, The Smurfit Institute, Trinity College, Dublin, Ireland *Corresponding author: SJ Martin, Molecular Cell Biology Laboratory, Department of Genetics, The Smurfit Institute of Genetics, Trinity College Dublin, Dublin D2, Ireland. Tel: + 3531896 1289; Fax: + 3531679 8558; E-mail: martinsj@tcd.ie
} 
immune responses by killing autoreactive lymphocytes, as well as dendritic cells that have failed to become fully differentiated.

Finally, the decision to mount an immune response can also be heavily influenced by whether antigen is encountered in the presence of apoptotic or necrotic cells. ${ }^{9}$ Much evidence now suggests that release of certain cellular constituents (collectively called 'danger signals' or 'alarmins'), as typically occurs during primary or secondary necrosis, can profoundly influence the activation of dendritic cells, which is critical to the initiation of most immune responses. Thus, the swift clearance of apoptotic cells by phagocytes can be instrumental in determining whether an immune response is initiated or not.

In the current issue of Cell Death and Differentiation, a series of reviews discuss some of the above aspects of immune function and the role of apoptosis as an influence upon, as well as an end point of, immune function. Opferman focuses primarily on the role of apoptosis in lymphocyte development and in particular the role of the Bcl-2 family in regulating death of $B$ and $T$ lymphocytes at different developmental stages. ${ }^{10}$ This is followed by a comprehensive account of NK-mediated activation and recognition of target cells by Moretta et al., ${ }^{11}$ and an overview of the detailed mechanistic aspects of CTL and NK granule-dependent killing. $^{12}$ Finally, attraction, recognition and clearance of apoptotic cells by phagocytic cells are addressed by Erwig and Henson. ${ }^{13}$

Munro also wrote that 'a little inaccuracy sometimes saves a ton of explanation'. Please forgive any little inaccuracies above for these reasons.

1. Jacobson MD, Weil M, Raff MC. Cell 1997; 88: 347-354.

2. Strasser A, Bouillet P. Immunol Rev 2003; 193: 82-92.

3. Van Parijs L, Abbas AK. Science 1998; 280: 243-248.

4. Strasser A, Pellegrini M. Trends Immunol 2004; 25: 610-615.

5. Bradley LM, Haynes L, Swain SL. Trends Immunol 2005; 26: 172-176.

6. Chen M et al. Science 2006; 311: 1160-1164.

7. Barry M, Bleackley RC. Nat Rev Immunol 2002; 2: 401-409.

8. Voskoboinik I, Smyth MJ, Trapani JA. Nat Rev Immunol 2006; 6: 940-952.

9. Matzinger P. Science 2002; 296: 301-305.

10. Opferman JT. Cell Death Differ 2008; 15: 234-242.

11. Moretta A, Marcenaro E, Parolini S, Ferlazzo G, Moretta L. Cell Death Differ 2008; 15 226-233.

12. Cullen SP, Martin SJ. Cell Death Differ 2008; 15: 251-262.

13. Erwig LP, Henson PM. Cell Death Differ 2008; 15: 243-250. 